\title{
Pregnancy Rates with Recombinant versus Urinary Human Chorionic Gonadotropin in In Vitro Fertilization: An Observational Study
}

\author{
József Zeke, ${ }^{1}$ Katalin Kanyó, ${ }^{1}$ Helga Zeke, ${ }^{1}$ Áron Cseh, ${ }^{2}$ Barna Vásárhelyi, ${ }^{2,3}$ \\ András Szilágyi, ${ }^{4}$ and János Konc ${ }^{1}$ \\ ${ }^{1}$ Infertility Center of Buda, Szt János Hospital, H-1125 Budapest, Hungary \\ ${ }^{2}$ Research Group of Pediatrics and Nephrology, Hungarian Academy of Sciences, \\ H-1083 Budapest, Hungary \\ ${ }^{3}$ Department of Laboratory Medicine, Semmelweis University, \\ $\mathrm{H}-1083$ Budapest, Hungary \\ ${ }^{4}$ Department of Obstetrics and Gynecology, Mór Kaposi University Teaching Hospital, \\ H-7400 Kaposvár, Hungary
}

Received 14 September 2011; Accepted 12 October 2011

Academic Editor: Takeshige Otoi

Randomized clinical trials (RCTs) demonstrated the equal efficacy of urinary human chorionic gonadotropin (uhCG) and recombinant hCG (rhCG) products in in vitro fertilisation (IVF). However, limitations inherent with RCTs necessitate the reinforcement of RCT results in real-life. We retrospectively analyzed pregnancies after treatment with rhCG and uhCG products $(n=391$, and 96, resp.). We found that laboratory-verified pregnancy occurred more frequently in rhCG patients than in those on uhCG $(43 \%$ versus $30 \%, P=0.02)$. The association remains significant $(P=0.002)$ after its adjustment for clinical characteristics. The prevalence of laboratory-verified pregnancies was higher with $\mathrm{GnRH}$ agonist use $(P=0.012)$ and $\mathrm{BMI}$ under $30 \mathrm{~kg} / \mathrm{m}^{2}(P=0.053)$ while decreased the age $(P=0.014)$ and the number of previous failed attempts $(P=0.08)$. Similar (but not significant) trends were observed with rates of pregnancy filled the 24th week. These results reinforce RCTs supporting the notion that rhCG is more efficient as uhCG during IVF.

KEYWORDS: urinary hCG, recombinant hCG, assisted reproduction, laboratory-verified pregnancy 


\section{INTRODUCTION}

The use of human chorionic gonadotropins (hCGs) to achieve final follicular maturation and triggering follicular rupture is well established in women undergoing ovulation induction.

During the spontaneous menstrual cycle, a preovulatory LH surge initiates the ovulation. However, in order to avoid premature rupture of follicles during in vitro fertilization, the spontaneous LH surge is prevented by the administration of GnRH agonists or antagonists and the ovulation is induced artificially. For ovulation induction, exogenous hCG is used due to its pharmacological effects on LH receptor [1].

hCGs in pharmacological preparations were originally derived from urinary samples of pregnant women. Urinary hCG (uhCG) products may have, however, great biological variability with a significant batch-to-batch variation due to their human origin. Therefore, recombinant technology has been introduced for the production of recombinant hCG (rhCG) with high-purity and batch-to-batch consistency. Other advantage of rhCG products over uhCG preparation is their availability in different dose strengths that provide an opportunity for individualized therapy. However, there is less experience with rhCG products in Hungary, particularly due to the fact that until 2008, the use of rhCG products in IVF was not reimbursed by the National Health Fund.

The equivalency between uhCG and rhCG preparations has been demonstrated by several well-designed randomized clinical trials (RCTs). In 2005, a Cochrane review analyzed seven RCTs, of those four compared rhCG and uhCG products. There was no statistically significant difference between rhCG and uhCG regarding the ongoing pregnancy/live birth rate [2]. Later on, these results were reinforced by further RCTs [3-6]. The current knowledge on the comparable efficacy of uhCG and rhCG relies, however, exclusively on RCT data. While RCTs are suitable to compare different therapeutic approaches in a wellcontrolled environment on a selected patient population, the results are not necessarily representative for the real life, where a more heterogenous patient population is treated without the strict monitoring required by study sponsors. Therefore, a noninterventional or observational approach to obtain data about rhCG or uhCG therapy may add valuable information on the efficacy of these products under routine IVF practice.

Our IVF centre in Szent János Hospital, Budapest, Hungary, treats about 1,000 suitable infertile women with IVF per year. In 2008, we started to switch to the regular use of rhCG instead of uhCG, as during that time the National Health Fund approved the reimbursement of rhCG. However, some colleagues resisted this change and used uhCG further. In our retrospective analysis, we took the opportunity and compared the IVF pregnancy outcomes (pregnancy verified by laboratory tests and pregnancy fulfilled 24th weeks of gestation) after administration of uhCG and rhCG products.

\section{PATIENTS AND METHODS}

In our retrospective analysis we collected data regarding the IVF procedures performed between February, 2008 and January, 2009 (start of rhCG reimbursement and retirement of colleagues preferring uhCG, resp.) (Figure 1).

Infertile couples were assessed and the suitability of the woman, for IVF was established in accordance with the Bourn Hall Guide to clinical and laboratory practice [7]. In eligible women multiple ovarian follicle development is achieved using the $\mathrm{GnRH}$ agonist long protocol or $\mathrm{GnRH}$ antagonist protocol combined with recombinant FSH.

In general, administration of GnRH agonist ( $0.1 \mathrm{mg}$ triptorelin s.c., Decapeptyl $0.1 \mathrm{mg} / 1 \mathrm{~mL}$, Ferring GmbH Germany) is introduced in the luteal phase of the previous cycle. After 10-12 days of GnRH agonist administration, once suppression is confirmed, stimulation with $225 \mathrm{IU}$ of recombinant FSH (Gonal-F; Merck-Serono, Geneva, Switzerland) or (Puregon Organon, Oss, Netherland) is started. The first ultrasound is performed on day 5 of stimulation. The dose of the gonadotropin can be increase or decrease at this point based on response. Ultrasound assessment and serum E2 measurements are used to monitor follicle growth.

In cases of GnRH antagonist protocols, the ovarian stimulation is performed using a recombinant FSH product (Gonal-F; Merck-Serono, Geneva, Switzerland). The initial gonadotropin dose is predefined 


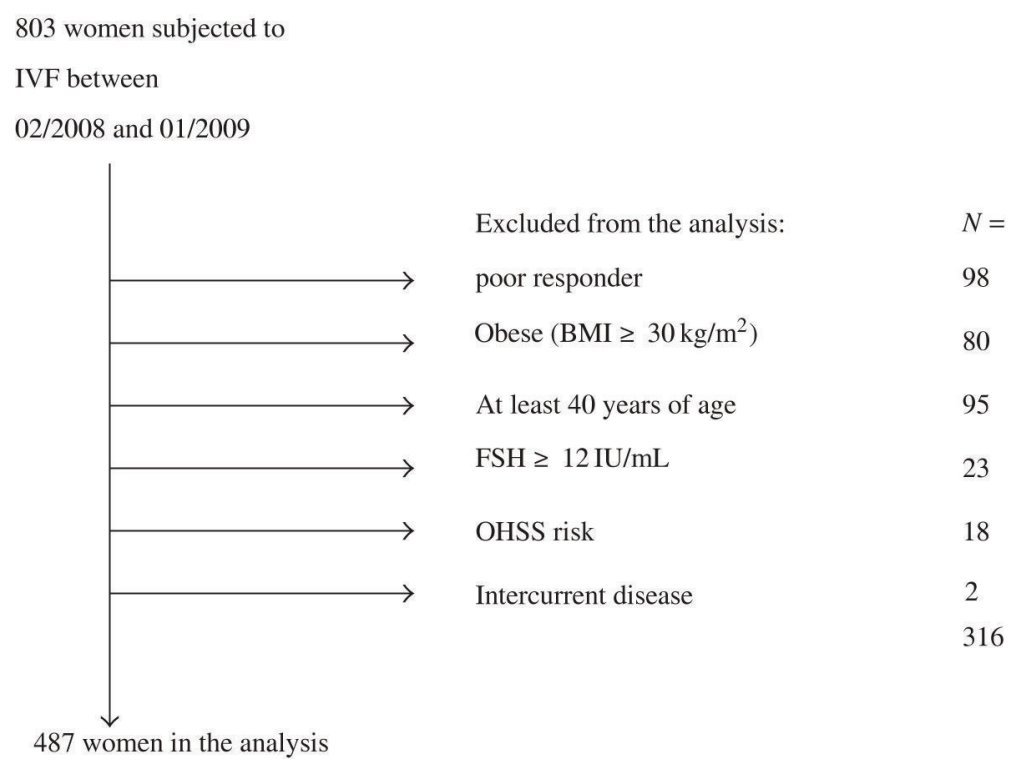

FIGURE 1: The selection of patients for our study.

at $225 \mathrm{IU}$ for all patients and is stable for 5 days; from this day onward, a $0.25 \mathrm{mg} / \mathrm{day} \mathrm{GnRH}$ antagonist is coadministered (Cetrotide; Merck-Serono, Geneva, Switzerland or Orgalutran; Organon, Oss, Netherland).

The decision whether to use a GnRH agonist or a GnRH antagonist is based on patients' preference and on the experience during prior IVF cycles with the same patient [8].

As three leading follicles reach $17 \mathrm{~mm}$ in diameter, rhCG or uhCG is administered to induce final follicular maturation. In general, the dose of rhCG depends on BMI, E2 levels on day of hCG administration, and the experience with previous IVF cycles. We give 6,500 IU, 9,750 IU, or 13,000 IU to the patients in an individualized manner, respectively. For uhCG, 10,000 IU is administered consistently.

Our effective institutional guideline authorizes our IVF experts to decide whether rhCG or uhCG are to be administered provided that the patient is under 40 years of age, is not severely obese (BMI $\left.\geq 30 \mathrm{~kg} / \mathrm{m}^{2}\right)$, her basal FSH levels are under $12 \mathrm{IU} / \mathrm{L}$, and presented poor response to prior stimulation challenges $(<3$ oocytes, cancelled cycle, etc.). (If these criteria are not fulfilled, rhCG products are preferred to uHCG as they allow individual dose titration.) Therefore, we limited our data collection to this population.

Transvaginal oocyte retrieval is scheduled 36 hours after the final hCG injection. The maturity of retrieved oocytes is assessed, then they are fertilised by conventional insemination or intracytoplasmatic sperm injection (ICSI). The success of fertilization is assessed the following day. The embryos are cultured for 3-5 days before implantation. Luteal support is administered in the form of $600 \mathrm{mg}$ micronized progesterone vaginally in divided doses starting after oocyte retrieval (Utrogestan Lab Besius, France).

Our retrospective data collection was approved by an institutional ethical committee and fulfills criteria of Helsinki declaration.

From patients' medical records, we obtained the following data: age, body mass index, number of previous attempts; endometrial thickness, basal FSH value, use of GnRH agonists or antagonists, and use dose of rhCG or uhCG. As outcome measures, pregnancy verified by laboratory tests and pregnancy fulfilled 24th weeks of gestation were used. In case of several attempts at the same patient $(n=3)$ during the analyzed period the data of the last attempt were used in our analysis.

Clinical characteristics in rhCG and uhCG groups were compared by $t$-test (continuous variables) or Chi-square test (dichotomic variables). The efficacy of rhCG and uhCG was first compared with Chisquare test. The association between rhCG/uhCG use and outcome was adjusted to age, body mass index, previous number of attempts, endometrium thickness, and use of GnRH agonist or antagonist using a logistic regression model. $P$ values under 0.05 were considered as statistically significant. 
TABLE 1: Patients' enrollment, clinical characteristics and outcomes. Abbreviations: rhCG: recombinant human chorionic gonadotropin; uHCG: urinary human chorionic gonadotropin; BMI: body mass index; $\mathrm{GnRH}$ : gonadotrop-releasing hormone; IU: international unit; g.w: gestational week.

\begin{tabular}{|c|c|c|c|}
\hline \multicolumn{4}{|c|}{ Clinical characteristics } \\
\hline & rhCG & uhCG & \\
\hline$n=$ & 391 & 96 & \\
\hline Age (years) & $32.5 \pm 3.5$ & $33.3 \pm 3.7$ & \\
\hline BMI $\left(\mathrm{kg} / \mathrm{m}^{2}\right)$ & $21.9 \pm 2.6$ & $22.5 \pm 2.6$ & \\
\hline No. of previous attempts & $1 \pm 1$ & $0.5 \pm 1$ & \\
\hline Endometrium thickness (mm) & $12 \pm 2$ & $11.5 \pm 2$ & \\
\hline \multicolumn{4}{|c|}{ Therapeutic characteristics } \\
\hline GnRH agonists & 159 & 46 & \\
\hline GnRH antagonists & 232 & 50 & \\
\hline 6500 IU dose & 100 & - & \\
\hline 9750 IU dose & 253 & - & \\
\hline $13000 \mathrm{IU}$ dose & 38 & - & \\
\hline \multicolumn{4}{|c|}{ Outcome measures } \\
\hline Pregnancy outcomes & & & Total: \\
\hline Laboratory verified pregnancy $(n=)$ & 169 & $29^{*}$ & 198 \\
\hline Pregnancy over 24th g.w $(n=)$ & 110 & 20 & 130 \\
\hline Laboratory verified pregnancy (\%) & $43 \%$ & $30 \%$ & $40.6 \%$ \\
\hline Pregnancy over 24th g.w (\%) & $28 \%$ & $21 \%$ & $26.7 \%$ \\
\hline
\end{tabular}

$P=0.021$, Chi-square-test.

\section{RESULTS}

During the analysed 12 months in 2008-2009, 803 IVF procedures were performed. Data of nonresponding, obese, elder women and those with FSH above $12 \mathrm{IU} / \mathrm{mL}$ were not included in the analysis (in total, $n=$ 296), as this population is skewed to the use of rhCG due to institutional guidelines. Signs and symptoms of suspected OHSS occurred in 15 on rhCG and in 3 patients on uhCG; the implantation was halted due to intermittent disease in 2 further patients. Finally, pregnancy data of 391 and 96 women on rhCG and uhCG, respectively, were compared (Table 1).

The clinical characteristics of both groups were comparable. Laboratory-verified pregnancy occurred more frequently in rhCG patients than in those on uhCG (odds ratio, OR [95\% confidence interval, CI]: 2.50 [1.40-4.35], $P=0.002)$. The association remained significant after its adjustment for clinical characteristics (Table 2). In addition to the benefit associated with the use of rhCG, GnRH agonist use also increased the occurrence of laboratory verified pregnancies, while in case of BMI, we observed a tendency (OR [95\% CI] 10.70 [10.12-20.58], $P=0.012$, and OR [95\% CI] 10.08 [0.99-10.17], $P=0.053$, resp.). However, the age correlated inversely and the number of previous attempts also tended inversely to the success (OR [95\% CI]: 0.93 [0.87-0.98], $P=0.014$, and OR [95\% CI] 0.87 [0.74-10.01], $P=0.080$, resp.).

No difference regarding the rates of pregnancy over 24th gestational week was observed between rhCG and uhCG groups by a Chi-square test. However, the association between the use of rhCG and pregnancy was almost significant $(P=0.056)$ in the logistic regression model when the contribution of GnRH agonists (associated with higher rates), age, and number of previous attempts (associated with lower rates) were also taken into account. 
TABLE 2: The impact of rhCG/uHCG use and clinical characteristics on the occurrence of laboratory-verified pregnancy and pregnancy over 24th week of gestation. Results of logistic regression analysis.

\begin{tabular}{|c|c|c|c|c|}
\hline & Odds ratio & \multicolumn{2}{|c|}{$95 \%$ confidence interval } & $P$ value \\
\hline \multicolumn{5}{|l|}{ Laboratory-verified pregnancy } \\
\hline rhCG & 2.50 & 1.40 & 4.35 & 0.002 \\
\hline Age & 0.93 & 0.87 & 0.98 & 0.014 \\
\hline GnRH agonist use & 10.70 & 10.12 & 20.58 & 0.012 \\
\hline BMI & 10.08 & 0.99 & 10.17 & 0.053 \\
\hline Number of previous attempts & 0.87 & 0.74 & 10.01 & 0.080 \\
\hline Endometrium thickness & 10.04 & 0.95 & 10.13 & 0.442 \\
\hline \multicolumn{5}{|l|}{ Pregnancy over 24th g.w } \\
\hline rhCG & 1.81 & 0.98 & 3.33 & 0.058 \\
\hline Age & 0.90 & 0.84 & 0.96 & 0.002 \\
\hline GnRH agonist use & 1.87 & 1.20 & 2.92 & 0.005 \\
\hline BMI & 1.00 & 0.91 & 1.09 & 1.000 \\
\hline Number of previous attempts & 0.83 & 0.69 & 1.01 & 0.067 \\
\hline Endometrium thickness & 1.04 & 0.95 & 1.15 & 0.410 \\
\hline
\end{tabular}

TABLE 3: Oocyte, MII, zygote, and embryo numbers with rhCG and uhCG products in assisted reproduction. Data are presented as median, interquartile range.

\begin{tabular}{lcc}
\hline & rhCG & uhCG \\
\hline Patient number & 391 & 96 \\
Oocyte number & $7(5-8)$ & $6(5-9)$ \\
MII number & $5(4-8)$ & $6(3.5-8)$ \\
Zygote number & $5(4-7)$ & $5(3-7)$ \\
Number of cleavaged embryos & $5(3-7)$ & $5(3-7)$ \\
Number of transferable embryos & $4(3-6)$ & $4(2-6)$ \\
\hline
\end{tabular}

No significant difference in oocyte numbers, MII numbers, zygote numbers, cleavaged embryo numbers, and transferable embryo numbers was obtained between rhCG- and uhCG-treated groups (Table 3).

\section{DISCUSSION}

In the analyzed time period, uhCG and rhCG preparations were used simultaneously in our institute for IVF procedures in women with low-risk for OHSS or resistance to ovulation induction. At this time personal preferences to the use of uhCG and rhCG preparations in this patient population differed, and this provided an opportunity for us to perform a direct comparison between these products. In our analysis, we did not aim to create a homogenous population; instead, we wanted to collect data regarding "real-life" patients. Therefore, the criteria for patients' enrollment were not strict and were used just to identify that high-risk population, where rhCG-preparations were already used in a routine manner.

The results of our observational study indicate that laboratory-verified pregnancy is positively associated with the use of rhCG with an about 13\% increase in its rate. In addition, a 7\% (nonsignificant) increase in rates of pregnancy fulfilling the 24th gestational week was also noted. As patients were heterogenous in term of clinical characteristics, we adjusted these observations to these factors, and these associations were maintained (and the positive effect of rhCG on ongoing pregnancy became also near 
significant). While our retrospective analysis did not allow to explore the causes, one should speculate that advantage in rhCG use compared to uhCG such as products' availability in different dose strengths and no batch-to-batch inconsistency may be translated to improved outcomes.

Some of the clinical and therapeutic factors that we adjusted for had also an impact on pregnancy outcomes. Of these, patients' age is well established [9] although it is important to emphasize that we did not enrolled women with $\geq 40$ years of age. The same applies for BMI; as we omitted obese subjects from our analysis, the documented adverse effect of obesity on IVF success could not be demonstrated [10, 11]. Instead, a rather positive association between BMI and laboratory-verified pregnancies was detected, indicating that higher, but still normal BMI (that is possibly associated with higher estrogen levels due to aromatase activity in adipose tissues [12]) is positively associated with outcome. The almost significant inverse association of pregnancies with the number of unsuccessful IVF cycles also supports current literary data [13].

Another important and striking observation of our work is the significant benefit of GnRH agonists compared to GnRH antagonists. This is in contrast to our previous observation [8] and also to the report of Bodri et al. who conducted a systematic review and concluded that ongoing pregnancy rate is similar with these drugs [14]. As the goal of our present analysis was to compare rhCG and uhCG preparations, we cannot explore the mechanism of this finding. As GnRH agonist and antagonist treatment depended on patient's preference and/or history with previous attempts, the use of this drug was equally distributed in rhCG and uhCG patients and was independent of patients' clinical characteristics. This finding, therefore, may indicate that (1) under real-life conditions, GnRH agonist and antagonist treatment is not equally effective, or (2) personal preference to GnRH agonist or antagonist is linked to some undetected feature (e.g., educational or socioeconomical status) that may contribute different results. Further targeted studies are required to elucidate this issue.

During the recent decade, several RCT-s have been published on the efficacy and suitability of rhCG in IVF. RCT-s have, however, inherent limitations due to strict patient enrollment criteria. Therefore, postmarketing studies are required to establish RCT results in real life. In our observational study, we detected significant advantage of rhCG over uhCG in terms of laboratory-verified pregnancy. Therefore, we consider that this retrospective study adds important information to the existing and extensive knowledge on the clinical efficacy of rhCG preparations during an IVF procedure.

\section{CONFLICT OF INTERESTS}

The authors report no financial or commercial conflicts of interest.

\section{ACKNOWLEDGMENT}

The authors are grateful to embryologist R. Kriston for her help in the data collection to this paper.

\section{REFERENCES}

[1] M. Ludwig, K. J. Doody, and K. M. Doody, "Use of recombinant human chorionic gonadotropin in ovulation induction," Fertility and Sterility, vol. 79, no. 5, pp. 1051-1059, 2003.

[2] H. G. Al-Inany, M. Aboulghar, R. Mansour, and M. Proctor, "Recombinant versus urinary human chorionic gonadotrophin for ovulation induction in assisted conception," Cochrane Database of Systematic Reviews, vol. 2, Article ID CD003719, 2005.

[3] A. Farrag, A. Costantini, C. Manna, and G. Grimaldi, "Recombinant HCG for triggering ovulation increases the rate of mature oocytes in women treated for ICSI," Journal of Assisted Reproduction and Genetics, vol. 25, no. 9-10, pp. 461-466, 2008. 
[4] P. Kovacs, T. Kovats, A. Bernard, J. Zadori, G. Szmatona, and S. G. Kaali, "Comparison of serum and follicular fluid hormone levels with recombinant and urinary human chorionic gonadotropin during in vitro fertilization," Fertility and Sterility, vol. 90, no. 6, pp. 2133-2137, 2008.

[5] E. G. Papanikolaou, C. Bourgain, H. Fatemi et al., "Endometrial advancement after triggering with recombinant or urinary HCG: a randomized controlled pilot study," Reproductive BioMedicine Online, vol. 21, no. 1, pp. $50-55,2010$.

[6] E. G. Papanikolaou, H. Fatemi, M. Camus et al., "Higher birth rate after recombinant hCG triggering compared with urinary-derived hCG in single-blastocyst IVF antagonist cycles: a randomized controlled trial," Fertility and Sterility, vol. 94, no. 7, pp. 2902-2904, 2010.

[7] A. Lass, "Investigation of the infertile couple for assisted conception," in A Textbook of In Vitro Fertilisation and Assisted Reproduction, P. R. Brinsden, Ed., chapter 2, pp. 13-26, Parthenone Publishing Group, Carnforth, UK, 1999.

[8] J. Zeke, K. Kanyó, R. Kriston, and E. Varga, "Experiences gained in IVF cycles using antagonist and long protocol," Magyar Noorvosok Lapja, vol. 69, no. 2, pp. 133-137, 2006 (Hungarian).

[9] Centers for Disease Control and Prevention website, AssistedReproductive Technology (ART) Report, http://apps.nccd.cdc.gov/art/FertilityClinicReport.aspx.

[10] M. L. Sneed, M. L. Uhler, H. E. Grotjan, J. J. Rapisarda, K. J. Lederer, and A. N. Beltsos, "Body mass index: impact on IVF success appears age-related," Human Reproduction, vol. 23, no. 8, pp. 1835-1839, 2008.

[11] A. Dokras, L. Baredziak, J. Blaine, C. Syrop, B. J. VanVoorhis, and A. Sparks, "Obstetric outcomes after in vitro fertilization in obese and morbidly obese women,” Obstetrics \& Gynecology, vol. 108, no. 1, pp. 61-69, 2006.

[12] L. R. Nelson and S. E. Bulun, "Estrogen production and action," Journal of the American Academy of Dermatology, vol. 45, no. 3, pp. S116-S124, 2001.

[13] M. K. Martin-Johnston, M. L. Uhler, H. E. Grotjan, A. S. Lifchez, J. M. Nani, and A. N. Beltsos, "Lower chance of pregnancy with repeated cycles with in vitro fertilization," Journal of Reproductive Medicine for the Obstetrician and Gynecologist, vol. 54, no. 2, pp. 67-72, 2009.

[14] D. Bodri, S. K. Sunkara, and A. Coomarasamy, "Gonadotropin-releasing hormone agonists versus antagonists for controlled ovarian hyperstimulation in oocyte donors: a systematic review and meta-analysis," Fertility and Sterility, vol. 95, no. 1, pp. 164-169, 2011.

\section{This article should be cited as follows:}

József Zeke, Katalin Kanyó, Helga Zeke, Áron Cseh, Barna Vásárhelyi, András Szilágyi, and János Konc, "Pregnancy Rates with Recombinant versus Urinary Human Chorionic Gonadotropin in In Vitro Fertilization: An Observational Study," TheScientificWorldJOURNAL, vol. 11, pp. 1781-1787, 2011. 


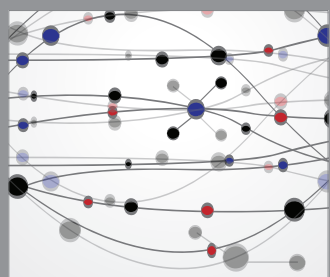

The Scientific World Journal
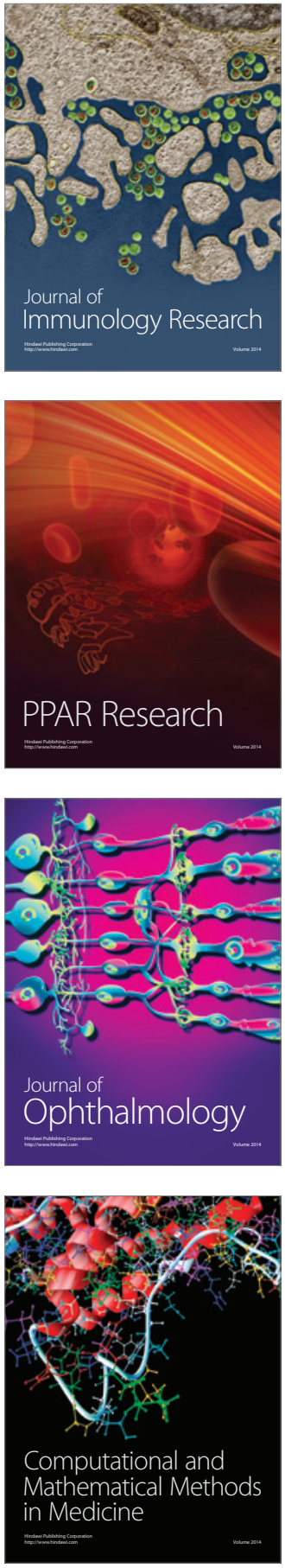

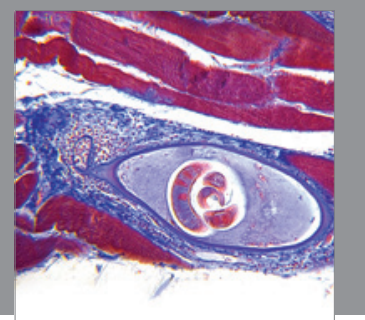

Gastroenterology

Research and Practice
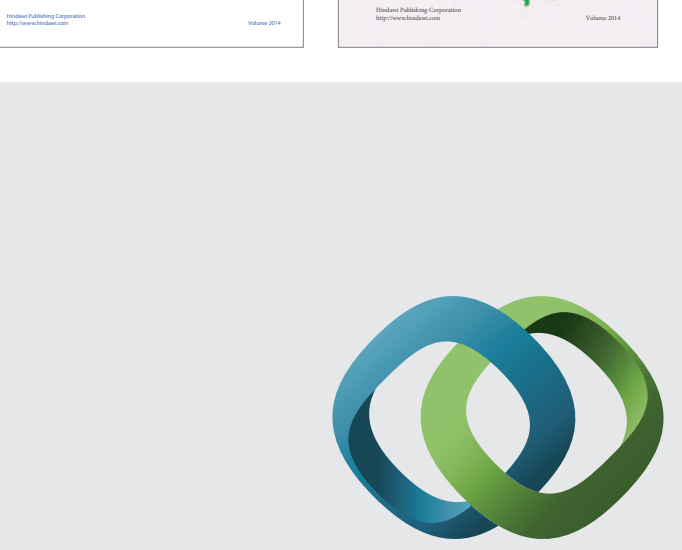

\section{Hindawi}

Submit your manuscripts at

http://www.hindawi.com
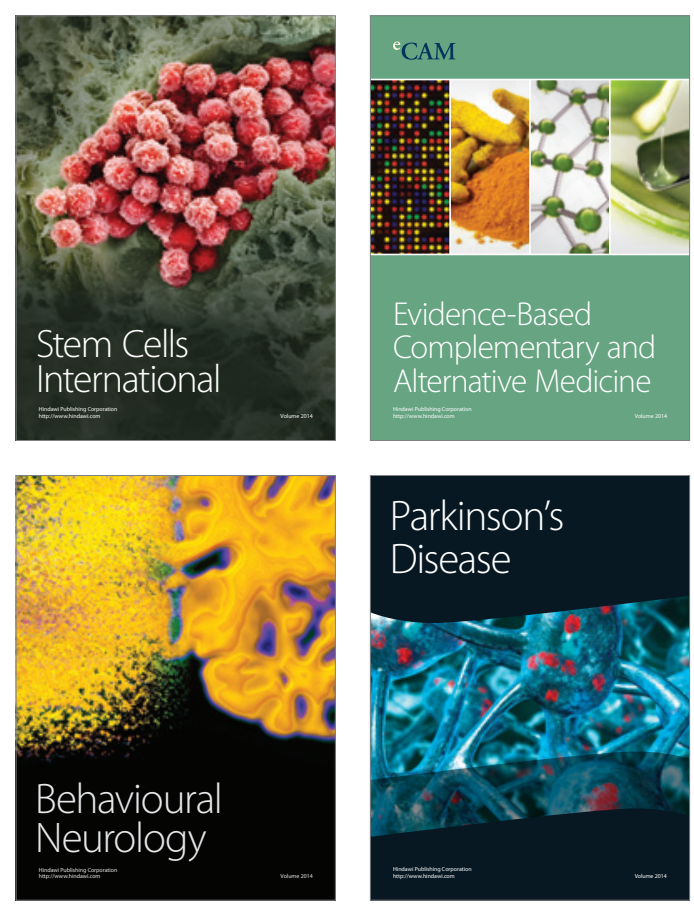

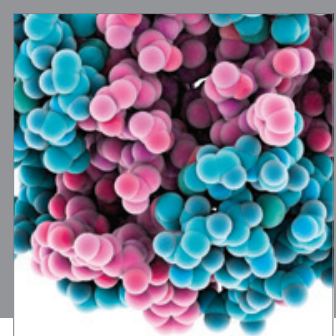

Journal of
Diabetes Research

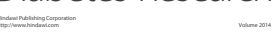

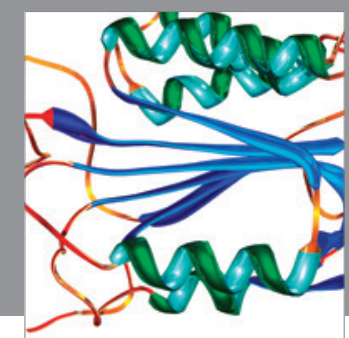

Disease Markers
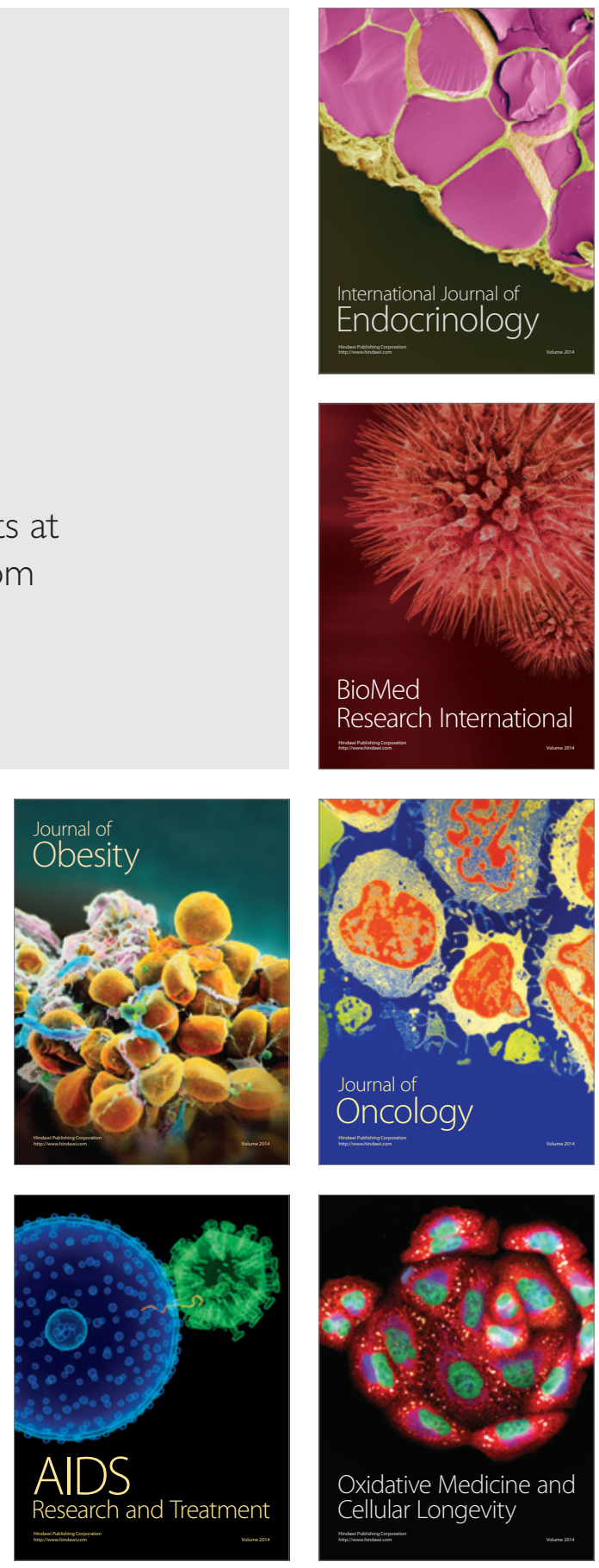